\title{
Synthesis of one-cycle ultra-short laser pulses for High-order Harmonic Generation
}

\author{
E. Neyra,${ }^{1}$ F. Videla,${ }^{1}$ M. F. Ciappina, ${ }^{2}$ J. A. \\ Pérez-Hernández, ${ }^{3}$ L. Roso, ${ }^{3}$ and G. A. Torchia ${ }^{1},{ }^{\text {, }}$ \\ ${ }^{1}$ Centro de Investigaciones Ópticas, \\ CONICET-CICBA-UNLP, Camino Centenario y 506, \\ M.B. Gonnet (1897), Pcia. Bs. As., Argentina \\ ${ }^{2}$ Institute of Physics of the ASCR, ELI-Beamlines project, \\ Na Slovance 2, 18221 Prague, Czech Republic \\ ${ }^{3}$ Centro de Láseres Pulsados (CLPU), Parque Científico, \\ E-37185 Villamayor, Salamanca, Spain
}

(Dated: March 26, 2018)

\begin{abstract}
We present a technique for the synthesis of ultra-short laser pulses with approximately one cycle (FWHM) of temporal duration. These pulses are characterized by a certain degree of chirp. We show that these pulses produce both an enhancement on the high-order harmonic generation (HHG) cutoff and a noticeable increase of the yield, when interact with an atomic system. Additionally, the asymmetric nature of the driven pulses plays an important role in the efficiency and cutoff extension of the high-order harmonics generated. Starting from the HHG spectra, we demonstrate it is possible to retrieve isolated attosecond pulses by spectral filtering. The analysis and interpretation of the different characteristic present in the HHG driven by this kind of pulses was carried out invoking classic arguments. Furthermore, a more complete description and validation of the HHG properties is performed by a quantum analysis, based on the integration of the time-dependent Schrödinger equation in full dimensionality (3D-TDSE).
\end{abstract}

PACS numbers: 42.65.Ky, 32.80.Fb, 32.80.Rm 


\section{INTRODUCTION}

In the last years, there has been a great advance in the generation of ultrashort laser pulses with stable carrier envelope phase (CEP). These systems can deliver sub-two optical cycles, with energies in the sub-millijoule regime, in the spectral range of $0.8-2 \mu \mathrm{m}$ [1 3 ]. On the

other hand, the synthesis of single-cycle and sub-cycle pulses was also achieved [4]. The interest in obtaining these kind of pulses mainly relies on the possibility of generating isolated attosecond pulses (IAP), which allow study of atomic and molecular electronic processes at their native temporal, attosecond, scale [7, 8]. The flagship for the generation of these pulses is the the well-known non linear strong field phenomena called high-order harmonic generation (HHG). HHG is one of the process that takes place when high intensity ultra-short laser pulses interact with atomic and molecular gaseous systems. This interaction generates a burst of high energy photons, typically in the XUV to soft-X-ray spectral range. These photons have odd frequencies of the original frequency, $\omega_{0}$, of the driving laser pulse [9-11].

A typical harmonic spectrum can be separated in three regions, namely (i) the perturbative one, where the low-order harmonics yield decays; (ii) a plateau, where all harmonics have the similar intensity, confirming the nonlinear nature of the process and (iii) an abrupt cutoff, at a maximum photon energy that can be calculated classically [9 11].

When a high intensity laser pulse interacts with an atom or molecule, it causes a deformation of the Coulomb potential and this enables the tunnel ionization of one or more electrons. Once in the continuum, the electrons are accelerated by the external electric field and driven back to recombine with the parent ion emitting their excess energy in a form of high energy photons. The maximum photon energy $E_{\text {cutoff }}$ is proportional to the $U_{p}$, the ponderomotive energy, that in turn can be written as $U_{p} \propto I \lambda^{2}$. Consequently, there are two possible routes to extend the harmonic cutoff, namely to increase (i) the laser wavelength or (ii) the laser intensity. However, a longer wavelength will imply an abrupt decreasing of the HHG efficiency, that scale as $\lambda^{-5.5}$ [14 17]. The reasons of this reduction can be traced out in the spreading of the electron wave-packet, because there is an increase of the trajectory duration $\tau \propto \lambda[14,15]$. In addition an increment in the intensity will also entail a loss of efficiency when the saturation intensity, characteristic of the target atom, ion or molecule, is exceeded [23 25]. Recently it was demonstrated a new avenue to extend the HHG cutoff driving the active media with a temporal and spatial synthesized field, although 
the experimental realization is still challenging [26, 27].

In this paper, we present a technique for the synthesis of ultrashort pulses joint with a theoretical analysis of its interaction with a noble gas. We focus our study in argon because it is one of the rare gas more commonly used in HHG experiments. Our approach allows obtaining chirped ultrashort pulses with approximately one cycle of temporal duration (FWHM). By starting with an optical pulse with central wavelength $\lambda_{0}$ and a temporal duration range from one and one a half cycle FWHM, we obtain optical pulses with $\lambda \approx$ $1.5 \lambda_{0}$. To perform this temporal synthesis, we superimpose two pulses with different degree of chirp. In order to achieve this goal it is not needed to employ a nonlinear medium; with a dispersive medium is enough. As we will see in the next sections, the instrumental parameter is the group velocity dispersion (GVD).

The theoretical study of the HHG driven by the synthesized pulses has been made by solving the time-dependent Schrödinger equation in full dimensionality (3D-TDSE) using argon as a target atom under the Single Active Electron (SAE) approximation. Additionally the classical analysis of the HHG characteristics is performed by solving the Newton-Lorentz equation.

The pulses synthesized by our technique have certain asymmetric characteristics in the pulse period. This means that the temporal region of the optical pulse when the electron is ionized is different from the one which enables it recombination. This asymmetry causes noticeable changes in the electron trajectory in comparison with a conventional sin-squared or gaussian pulse. A clear increase of the electron recombination energy is obtained, depending on the kind of asymmetry in the pulse. This, in turn, translates in an extension of the HHG cutoff. Besides, the electron travel time in the continuum will be different and this indeed improves the efficiency of the HHG, as it was mentioned before. In previous contributions, some authors proposed different ways of obtaining this asymmetry in one period of the optical pulses e.g. using the so-called perfect wave, summing up pulses with same and different colors, amongst others [18 22, 28, 29]. In these articles, a cutoff extension of the HHG spectrum and/or an enhancement in the harmonic yield are obtained depending on the synthesized pulse. In our approach, both of the mentioned effects in the HHG spectrum are achieved. 


\section{METHODS}

\section{Pulse Synthesis}

For the synthesis of the pulses we propose the experimental setup depicted in Fig. 1(a). In this scheme, we achieve the sum of two pulses with different degree of chirp (GVD) and a $\pi$ phase change between of them. In summary, we present the synthesis of four pulses varying the sign and degree of chirp of the initial pulses A and B.
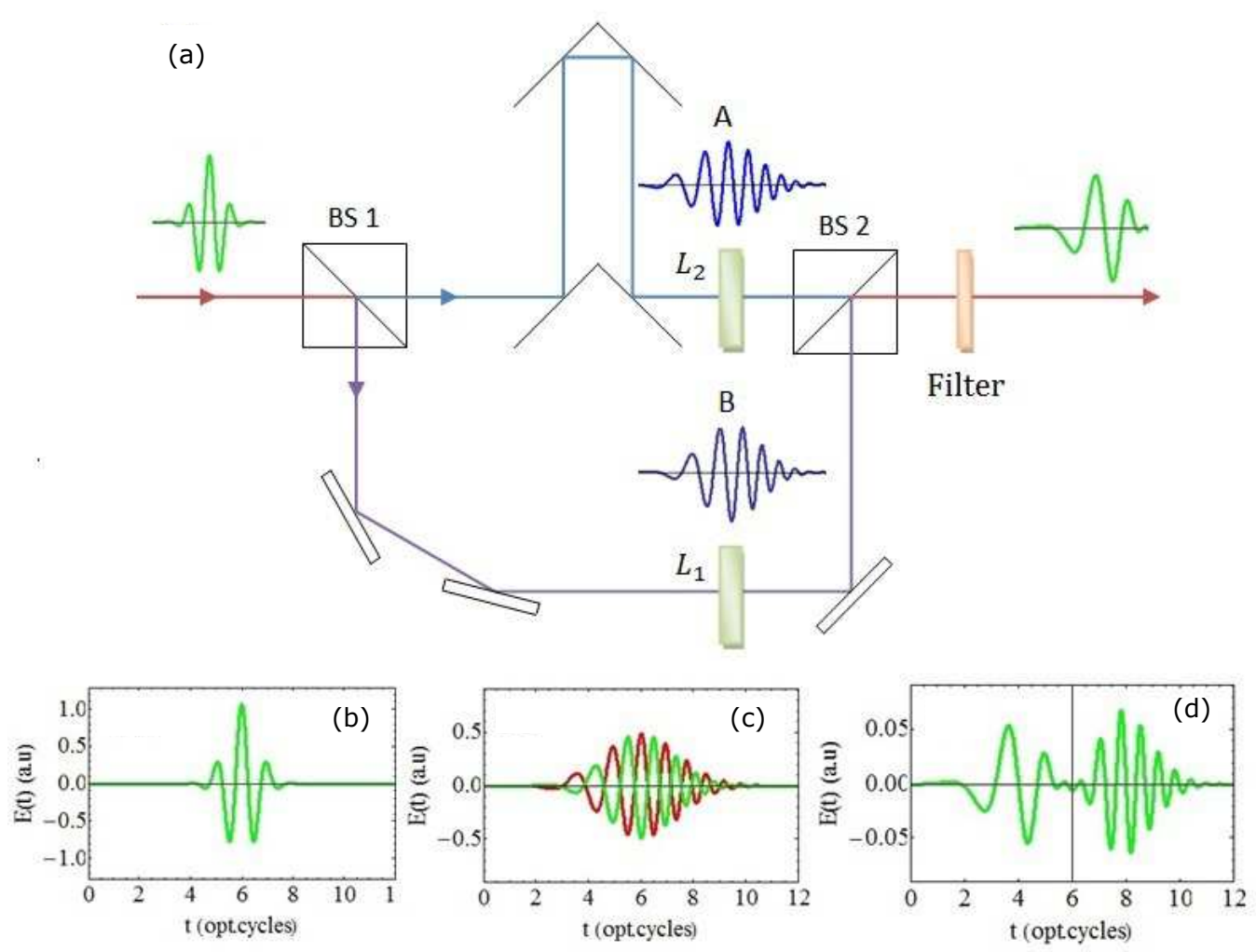

FIG. 1: Potential experimental set-up to carry out the proposed pulse synthesis.

Initially we have a gaussian pulse with a central wavelength $\lambda_{0}$ and one-cycle of FWHM [Fig. 1(b)]. This pulse is divided in two pulses, pulse A (PA) and pulse B (PB), through a beam splitter. PB crosses a dispersive media with length $L_{1}$ and is reflected by a number of odd mirrors. On the other hand, PA crosses a dispersive medium with length $L_{2}$ and travels through the same optical path, but the mirrors that reflects it is a even number. This originates then a phase change in $\pi$ with respect to PB. Next, the two pulses merge 
in a beam splitter. The representation of these pulses superposition is showed in Fig. 1(c). Furthermore, in Fig. 1(d) we depict the temporal sum of these pulses. As it can be seen, we obtain a new pulse, that has two distinct spectral zones, each of them with a different wavelength. One of these regions has an approximate wavelength $\lambda \approx 1.5 \lambda_{0}$ and a one-cycle of FWHM, while the other one has a shorter wavelength, irrelevant for our study (note that it may be important for other applications). For this reason, we propose an optical filter to remove this higher frequencies region [Fig. 1(a)].
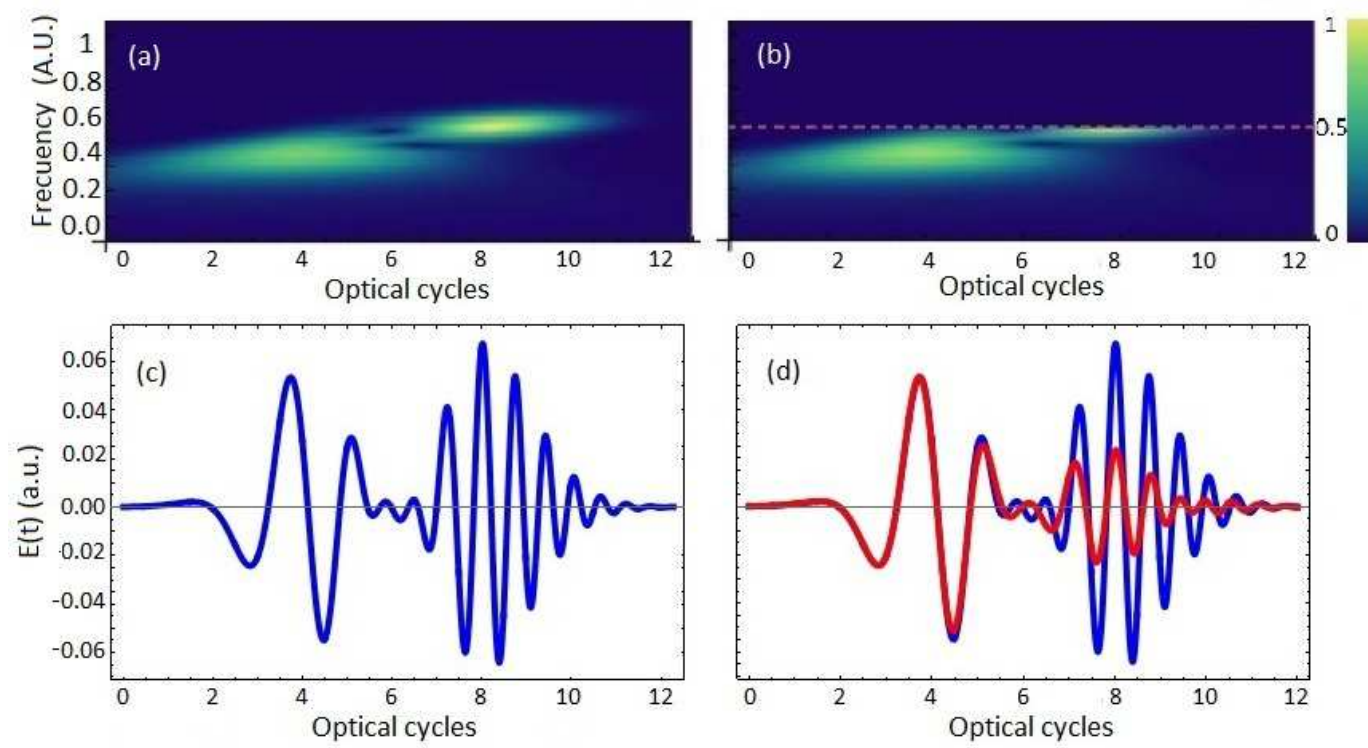

FIG. 2: (a) and (b) wavelet analysis of the synthesized and filtered pulses, respectively; (c) and (d) temporal profile of the synthesized and filtered pulse (in red), respectively.

Considering the broad spectral range of the two pulses, they are few femtoseconds FWHW long, and their clear temporal separation [Fig. 1(d)], the wavelet analysis of the synthesized pulse is a good choice in order to understand the effect of the proposed ideal optical filter. In Fig. 2(a) we show the wavelet analysis of the synthesized pulse [Fig. 2(c)]. In this case two regions are clearly observed, each temporarily centered, at four and eight optical cycles respectively. As it is expected, these two regions show different spectral content. The optical filter then was applied in order to keep the total spectral composition over the pulse at low frequencies, as it is shown in Fig. 2(b). The Fig. 2(d) shows in red dashed line the pulse obtained after crossing the ideal optical filter and in blue the original pulse. As it is observed of the figure, the filtered pulse does not change its form in the first part (which is of our interest), but in the second one its amplitude decreases dramatically. We demonstrated in a 
previous work that the second part of the pulse has no influence in the HHG process, since its amplitude is not enough to ionize the atom [19].

After the high frequency region removal, the four synthesized pulses are shown in Fig. 3. Each of those pulses was obtained by varying the length $L_{1}$ and $L_{2}$ and the sign of the chirp (up-chirp and down-chirp). The dispersive medium used was an SF14 glass [19]. In order to achieve the pulses 1 and 2 [Figs. 3(a) and 3(b)], the lengths of the media were $L_{1}=25 \mu \mathrm{m}$ and $L_{2}=30 \mu \mathrm{m}$ and the pulse 1 is up-chirped meanwhile the pulse 2 is downchirped. Likewise, the lengths of the media to obtain pulses 3 and 4 [Fig. 3(c) and 3(d)] were $L_{1}=20 \mu \mathrm{m}$ and $L_{2}=30 \mu \mathrm{m}$. Here pulse 3 and 4 are up- and down-chirped, respectively. In this representation the second part ( $t>6$ optical cycles) of the optical wave for each case was removed, since it has no important effects in the HHG.
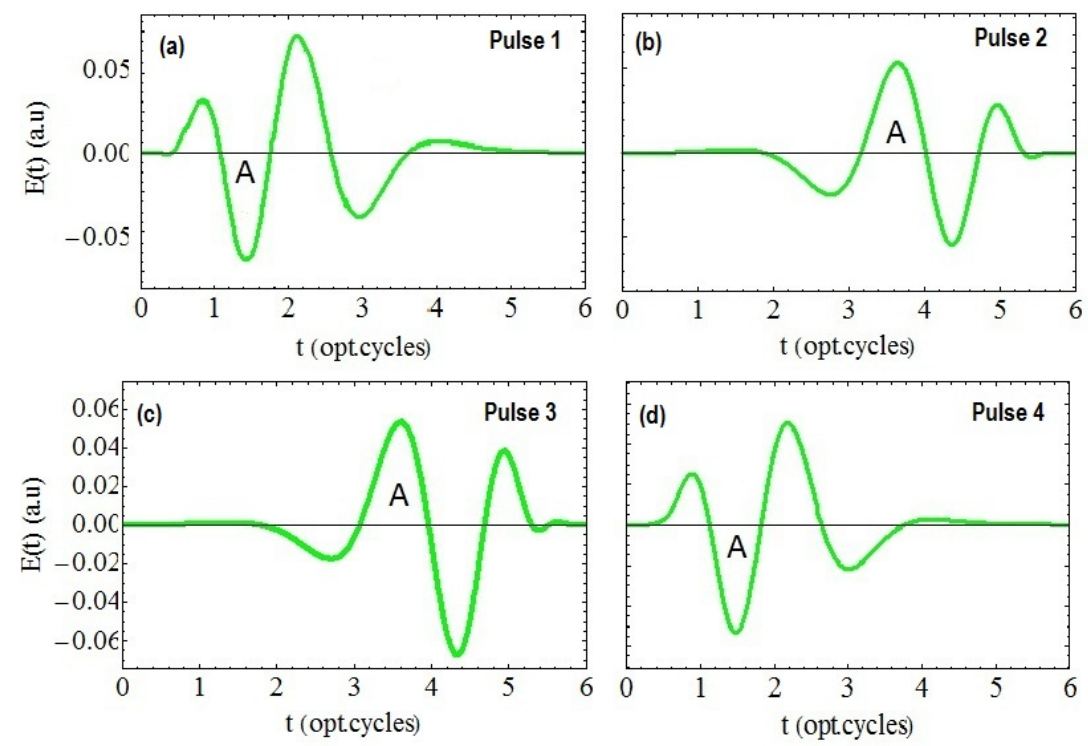

FIG. 3: Temporal profile of the four pulses synthesized in the present study. The pulse 1 (panel a) is obtained with $L_{1}=25 \mu \mathrm{m}, L_{2}=30 \mu \mathrm{m}$ and it is up-chirped; the pulse 2 (panel b) with $L_{1}=25 \mu \mathrm{m}, L_{2}=30 \mu \mathrm{m}$ and down-chirped; the pulse 3 (panel c) with $L_{1}=20 \mu \mathrm{m}$, $L_{2}=30 \mu \mathrm{m}$ and up-chirped and the pulse 4 (panel d) with $L_{1}=20 \mu \mathrm{m}, L_{2}=30 \mu \mathrm{m}$ and down-chirped. Note that the temporal region corresponding to higher frequencies was removed ( $t>6$ optical cycles). 


\section{Theoretical Methods}

According to the three-step model [11 13] the maximum photon energy, $E_{\text {cutoff }}$, in every harmonic spectrum is given by the semiclassical cut-off law,

$$
E_{\text {cutoff }}=I_{p}+3.17 U_{p}
$$

(atomic units are used throughout this paper unless otherwise stated) where $I_{p}$ is the ionization potential of the corresponding atom or ion target (in this work we focus our study on the argon atom, $I_{p}=0.57$ a.u., i.e. $\left.15.7 \mathrm{eV}\right), \omega_{0}$ is the central laser frequency and $U_{p}$ is the ponderomotive energy given by:

$$
U_{p}=\frac{E_{0}^{2}}{4 \omega_{0}^{2}}
$$

with $E_{0}$ being the laser electric field peak amplitude. As we will see in the next section, by the integration of the Newton-Lorentz equation it is possible to obtain the time dynamics of the laser-ionized electron, as well as to estimate the classical electron kinetic energy as a function of the ionization and recombination times.

In order to complement the semiclassical analysis we compute the HHG spectra generated by the synthesized pulses of Fig. 3 by numerical integrating the 3D-TDSE in the length gauge within the dipole approximation. As it is well known the harmonic yield of an atom is proportional to the Fourier transform of the dipole acceleration of its active electron. The latter can be calculated from the time propagated electronic wave function. We have used our code which is based on an expansion in spherical harmonics, $Y_{l}^{m}$, considering only the $m=0$ terms due to the cylindrical symmetry of the problem. The numerical technique to solve the 3D-TDSE is based on a Crank-Nicolson method implemented on a splitting of the time-evolution operator that preserves the norm of the wave function. Here, we attract our studies on the argon atom. This is so, considering this gas is one of the most commonly used in current HHG experiments. However, without loss of generality, the results explored in the present contribution can be extended to the rest of the noble gases. Hence, we have considered in our 3D-TDSE code the atomic potential reported in 30] to accurately describe the level structure of the argon atom under the SAE approximation. In addition, and in

order to interpret the detailed spectral and temporal behaviour of the HHG spectra, we perform a time-frequency analysis by means of a wavelet transform [31 33]. 


\section{RESULTS AND DISCUSSION}

The initial pulse before the synthesis is a gaussian pulse with one-cycle FWHM and a laser wavelength $\lambda_{0}=800 \mathrm{~nm}$ [see Fig. 1(b)]. For the proposed pulse synthesis there is no particular laser intensity requirements, since a non-linear medium is not necessary. Each of the four synthesized pulses reach the argon atom ionization threshold at a laser electric field $E_{0}=0.053$ a.u. (see point A, Fig. 3). This value corresponds to a laser intensity $I=10^{14}$ $\mathrm{W} / \mathrm{cm}^{2}$ and this is the value used in our simulations.

In Fig. 4 we show the HHG spectra that result from the interaction of argon atoms with the 4 synthesized pulses plotted in Fig. 3. In all the cases we numerically integrate the 3D-TDSE under the SAE (red lines). The HHG spectra of Figs. 4(a)-4(d) correspond to the pulses of Figs. 3(a)-3(d), respectively. Additionally, in Fig. 4(a) we include the HHG spectrum corresponding to a laser wavelength $\lambda_{0}=800 \mathrm{~nm}$ and intensity of $10^{14} \mathrm{~W} / \mathrm{cm}^{2}$ (black line). Finally, in Figs. 4(a)-4(d) the HHG spectra using "equivalent gaussian pulses" (blue lines) are included (see below for details).

In each spectrum can be observed a noticeable increase of the cutoff, compared to the one obtained with a conventional gaussian pulse with $\lambda_{0}=800 \mathrm{~nm}$ and the same intensity. This result is expected since the synthesized pulses have approximately a wavelength of $\lambda \approx 1.5 \lambda_{0}$ corresponding to $\lambda=1200 \mathrm{~nm}$. However, pulses 1 and 3, have a cutoff extension that clearly exceeds the cutoff given by a pulse with a wavelength $\lambda=1200 \mathrm{~nm}$. A possible explanation of the above phenomenon can be traced out by considering the change in the electron trajectories in relation to those developed when a pure sine-like pulse is used. Our proposed synthesis generate chirped pulses, characterized for certain degree of asymmetry in the temporal regions where the electron is ionized and recombines. This behaviour was studied in previous works, where asymmetric pulses are obtained using different techniques [18, 22, 28].

Additionally, we analyse the HHG yield for each of the four proposed synthesized pulses. In order to interpret the asymmetry effects, we introduce a series of "equivalent gaussian pulses" with a comparable wavelength, whose effect is to develop a similar HHG cutoff. Their associated HHG spectra are plotted in Figs. 4(a)-(d) (blue lines). It can be observed that all synthesized pulses have larger HHG yields than the equivalent guassian ones. Pulses 1 and 3, the ones that produce larger cutoffs, show one order of magnitude increase in the harmonic yield compared to their equivalent pulses counterparts. Note that all spectra 

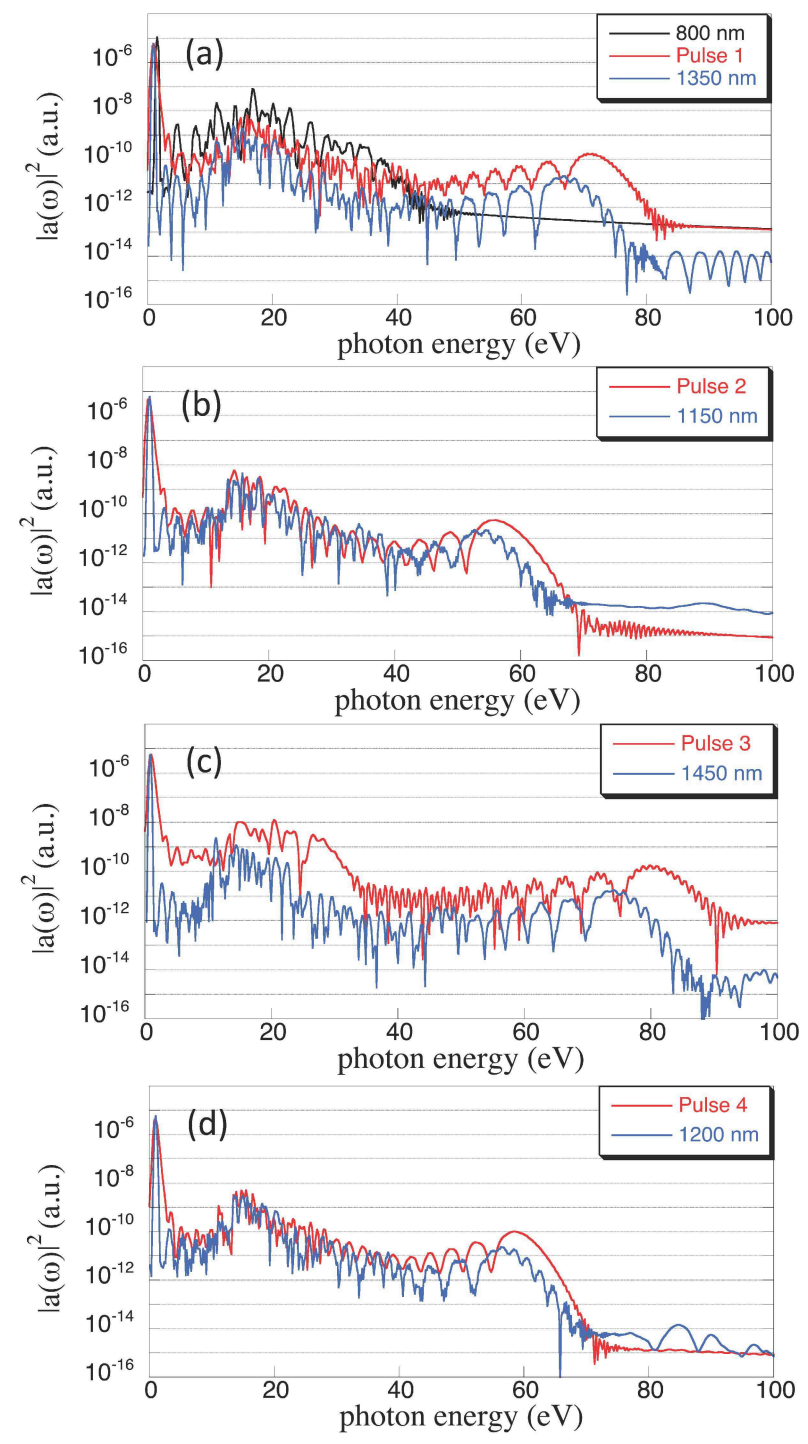

FIG. 4: 3D-TDSE HHG spectra in argon driven by the four synthesized pulses of Fig. 3 (red lines). Panel (a), pulse 1 [Fig. 3(a)], panel (b), pulse 2 [Fig. 3(b)], panel (c), pulse 3 [Fig. 3(c)] and panel (d), pulse 4 [Fig. 3(d)]. In Fig. 4(a) the HHG spectrum (black line) for a conventional gaussian pulse is included. Additionally, in all the panels the HHG spectra (blue line) of their "equivalent pulses" are represented (see the text for details).

The laser intensity is the same for all the cases, $I=10^{14} \mathrm{~W} / \mathrm{cm}^{2}$. The valley in the respective spectra around the $40 \mathrm{eV}$ is due to the argon Cooper minimum [34].

presents a valley in the yield around $40 \mathrm{eV}$ due to the argon Cooper minimum [34].

It is known that the HHG efficiency depends on the trajectory duration $(\tau)$ [14, 15]. As a consequence, in order to explain the higher yield observed in the HHG spectra when the argon atoms are driven by the synthesized pulses, we study the travel times of the electrons in 

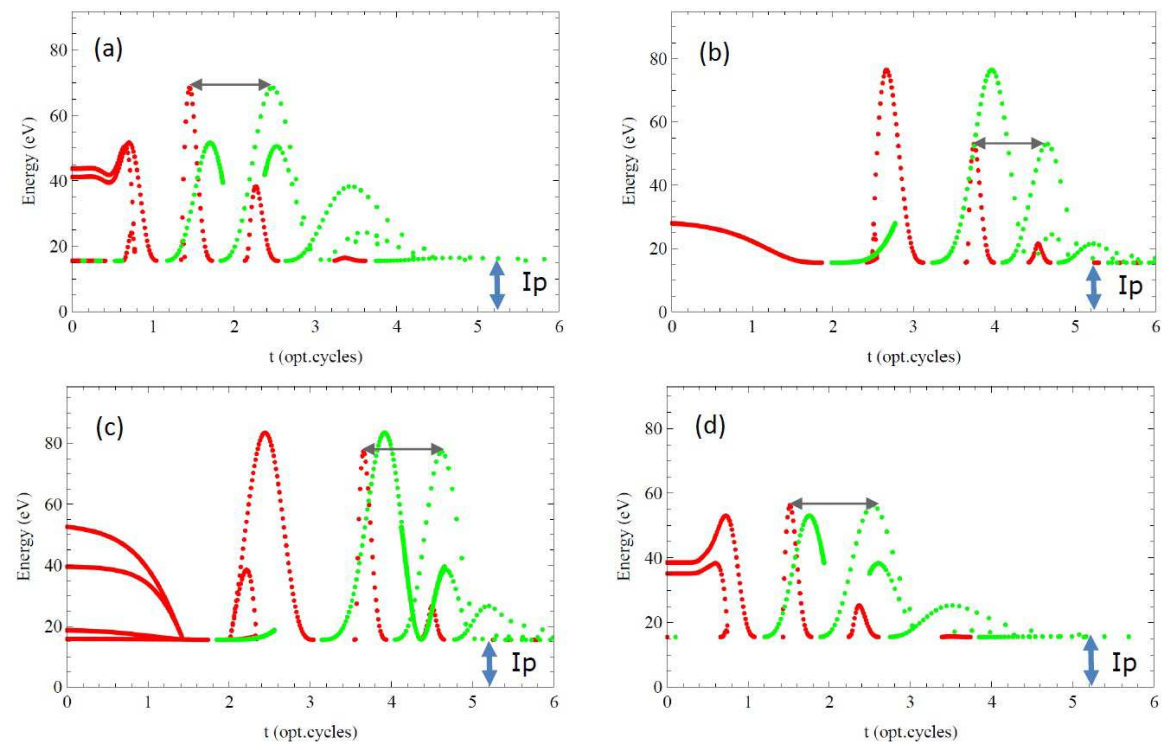

FIG. 5: Classical total electron energies (in eV) as a function of ionization (red points) and recombination (green points) times for the 4 synthesized pulses of the Fig. 3. The black arrows show the travel times of the electron trajectories at the cutoff energy. The $I_{p}(15.7$ $\mathrm{eV}$ ) of the argon atoms is displayed with blue arrows (note that one optical cycle is $2.66 \mathrm{fs}$ ).

\begin{tabular}{|c|c|c|}
\hline$\tau(\mathrm{fs})$ & synthesized pulses & equivalent pulses \\
\hline Pulse $\mathbf{1} \equiv \mathbf{1 3 5 0} \mathbf{~ n m}$ & $\mathbf{2 . 7 0} \mathbf{f s}$ & $\mathbf{3 . 2 9} \mathbf{f s}$ \\
\hline Pulse $2 \equiv 1150 \mathrm{~nm}$ & $2.33 \mathrm{fs}$ & $2.80 \mathrm{fs}$ \\
\hline Pulse $\mathbf{3} \equiv \mathbf{1 4 5 0} \mathbf{~ n m}$ & $\mathbf{2 . 3 8} \mathbf{f s}$ & $\mathbf{3 . 5 3} \mathbf{f s}$ \\
\hline Pulse $4 \equiv 1200 \mathrm{~nm}$ & $2.68 \mathrm{fs}$ & $2.93 \mathrm{fs}$ \\
\hline
\end{tabular}

TABLE I: Travel times for electrons at the cutoff energy, for both the synthesized and equivalent pulses, respectively.

the continuum through a classical analysis. In Fig. 5 we show the classical total energies as a function of ionization (red points) and recombination (green points) times (in optical cycles) for the 4 synthesized fields of Fig. 3. A quick inspection allows one to confirm the excellent correspondence between the classical cutoff energies and their quantum counterparts (see Fig. 4).

We perform a study comparing the travel times for the electron trajectories at the cutoff 
for both the synthesized and equivalent pulses. The former are extracted from the classical calculations (see the arrows in Fig. 5), meanwhile the latter are calculated as $\tau_{G}=0.75 T_{p}$ where $T_{p}=\frac{2 \pi}{\omega}$ is the period of a pure sine wave with $\omega=\frac{2 \pi c}{\lambda}$ ( $c$ is the speed of light). In table 1 we present the numerical values of the travel times $(\tau)$ corresponding to the cases plotted in Fig. 5. We highlight the travel times of pulses 1 and 3, because in these cases longer cutoff energies and better efficiency is observed. It is shown that all travel times values for the synthesized pulses are smaller than those of the equivalent pulses. As a consequence, a higher HHG efficiency should be expected, considering shorter travel times imply, in turn, less spread electron wavepackets and thus larger recollision probabilities.

On the other hand, in order to confirm the previous results and clarify some aspects of the HHG process driven by these synthesized pulses, we perform a wavelet analysis of the HHG spectra of Fig. 4. We present the results in Fig. 6. From these plots we appreciate the temporal region in which the high-order harmonics are emitted. In Fig. 6 (a) it is shown the wavelet analysis for black harmonic spectra (Fig. 4 (a)) which correspond to the pure gaussian pulse centred at $800 \mathrm{~nm}$ of wavelength. Figs. 6 (b) and 6(d) show three bursts of XUV radiation meanwhile Figs. 6(c) and 6(e) present only two. As a consequence, the attosecond pulses associated with these burst will have different spectral and temporal characteristics. In addition, the classical electron energies at recombination are superimposed in the same plots (solid black lines). As observed, the classical results are in excellent agreement with the quantum mechanical ones.

As corollary we present the synthesis of attosecond pulses using the HHG spectra that have larger cutoff and higher efficiency, i.e. the ones corresponding to pulses 1 and 3 [Figs. 3(a) and 3(c)].

In order to obtain an isolated attosecond pulse (IAP) we use a $20 \mathrm{eV}$ bandwidth filter close to the cut-off region, where only one event of electron recombination takes place. We show in Figs. 7(a) and 7(b) the HHG spectra joint with a shadow region that represents the filter. In Figs. 7(c) and 7(d) we show the IAPs obtained. As it is shown, from the pulse 3 is synthesized an IAP considerably shorter (260 as) than the IAP obtained from the pulse 1 (490 as). 

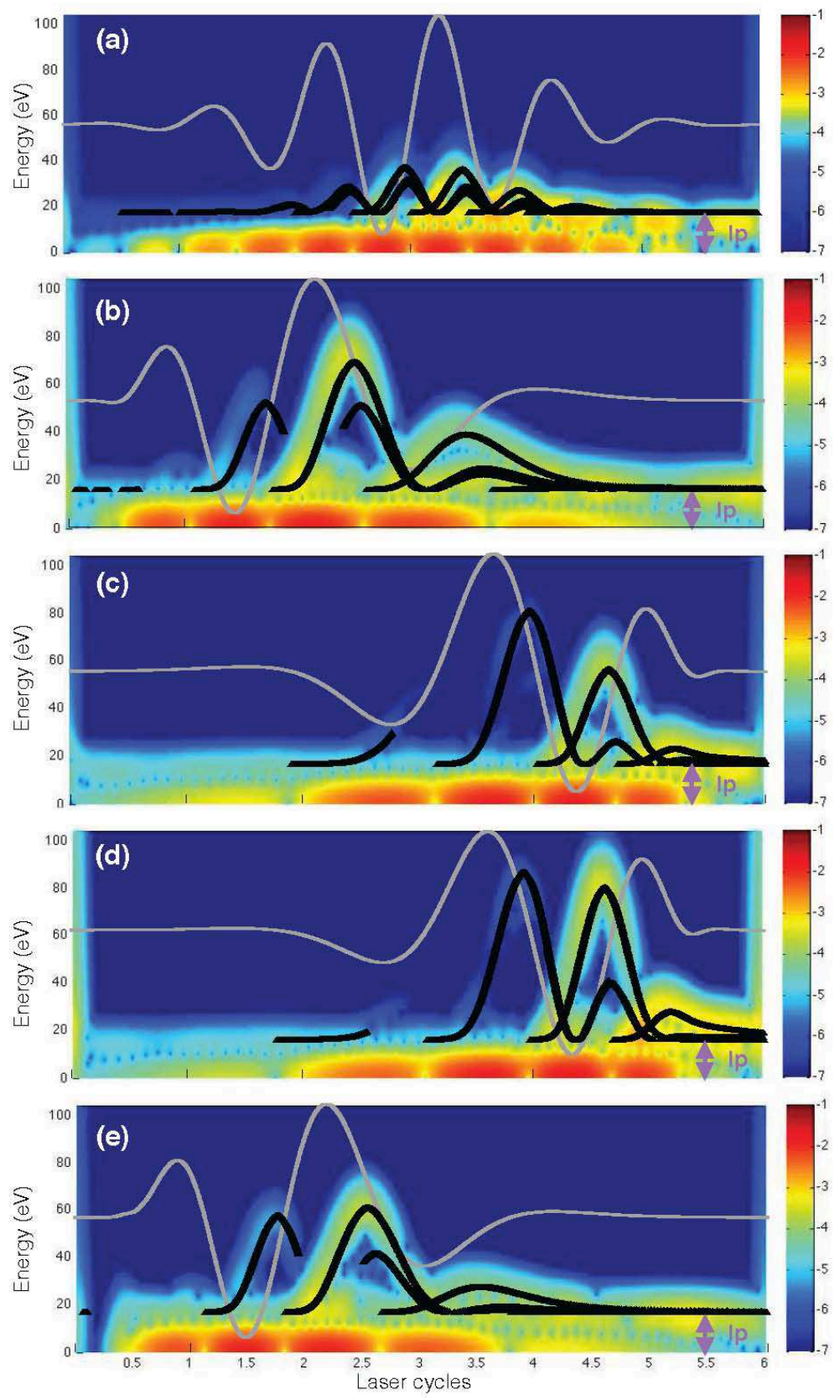

FIG. 6: Time-frequency analysis of the HHG spectra of Fig. 4. The classical electron energies at the recombination time is superimposed (solid black lines). The driving pulses are also plotted (solid gray line). The laser intensity is the same for all the cases, $I=10^{14}$

$$
\mathrm{W} / \mathrm{cm}^{2} \text {. }
$$

\section{CONCLUSIONS AND OUTLOOK}

We have proposed a new experimental approach to synthesize an approximately one-cycle laser pulse with a wavelength of $\lambda \approx 1.5 \lambda_{0}$, starting from a pulse with wavelength of $\lambda_{0}$. We 
(a)

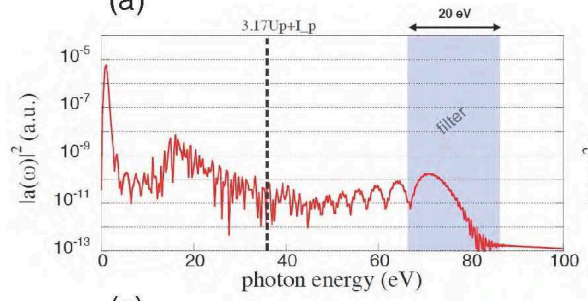

(c)

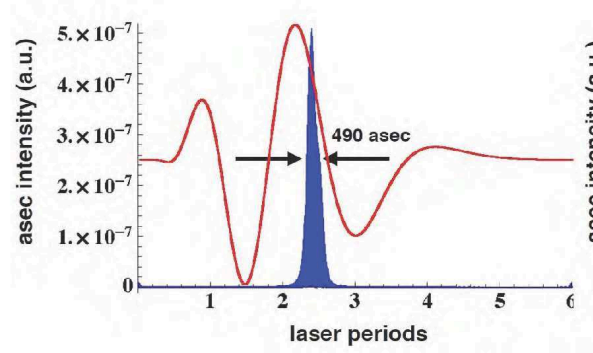

(b)

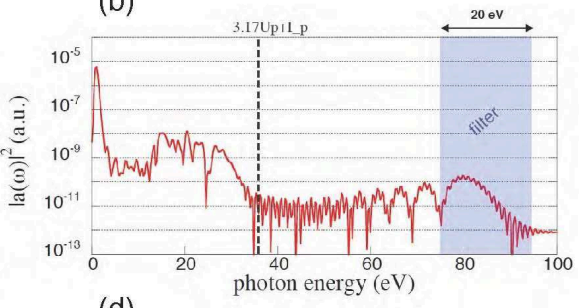

(d)

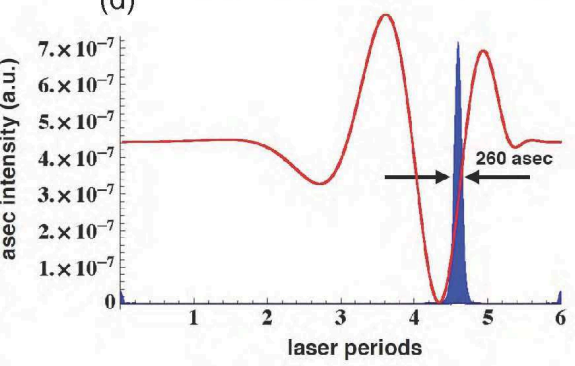

FIG. 7: HHG generated by the pulse 1 (a) and by the pulse 3 (b) and the corresponding attosecond pulses (c) and (d) respectively, using a $20 \mathrm{eV}$ filter in the cutoff region.

analyse four synthesized pulses with different characteristics and certain degree of chirp, all of them being obtained by varying the lengths $L_{1}$ and $L_{2}$ of a dispersive material, as well as the sign of its GVD.

We study the HHG process in argon atoms driven by those pulses, both by solving the Schrödinger equation in full dimensionality (3D-TDSE) and the classical analysis. All synthesized pulses give rise to an extension of the cutoff energy and a larger efficiency of the harmonic yield.

In order to compare the efficiency of the harmonics yield we introduce four equivalents pulses that produce the same cutoffs than the four synthesized pulses. We show that the HHG yields using the synthesized pulses have an efficiency one order of magnitude larger than their equivalent ones counterparts. By carefully studying the travel times of electrons in the continuum through the classical analysis of the ionization-recombination energies we are able to explain the reasons of this behavior in the HHG spectra.

In summary, we show a possible experimental setup to synthesize an approximately onecycle optical pulse to enhance both the HHG cutoff and yield. These improvements can be understood considering the synthesized pulses have a certain degree of asymmetry in the ionization-recombination temporal region. This introduces noticeable changes in the electrons trajectories when compared with a pure sine pulse. 


\section{ACKNOWLEDGEMENTS}

This work was partially supported by the Agencia Nacional de Promocin Cientfica y Tecnolgica (Argentina) under project PICT-2016-4086. This work was supported by the

project ELI - Extreme Light Infrastructure - phase 2 (CZ.02.1.01/0.0/0.0/15 008/0000162) and the project Advanced research using high intensity laser produced photons and particles (CZ.02.1.01/0.0/0.0/16 019/0000789) both from the European Regional Development Fund. J. A. P.-H. and L. Roso acknowledge Spanish Ministerio de Economía, Industria y Competitividad (MINECO) through the PALMA Grant No. FIS2016-81056-R, from LaserLab Europe IV Grant No. 654148, and from the Regional Government, Junta de Castilla y León, Grant No CLP087U16.

* gustavot@ciop.unlp.edu.ar

[1] B. E. Schmidt et al., Appl. Phys. Lett. 96121109 (2010).

[2] S. Driever et al., Appl. Phys. Lett. 102, 191119 (2013).

[3] D. Brida et al., J. Opt. 12, 013001 (2010).

[4] H. Shu-Wei et al., Nat. Phot. 8, 475-479 (2011).

[5] J. A. Cox et al., Opt. Lett. 17, 3579-3581 (2012).

[6] C. Manzoni et al., Laser Photonics Rev. 2, 129-171 (2015).

[7] G. Sansone et al., Science 314, 443-446 (2006).

[8] F. Krausz and M. I. Stockman, Nat. Phot. 8, 205-213 (2014).

[9] A. L'Huillier and P. Balcou, Phys. Rev. Lett. 70774 (1993).

[10] J. J. Macklin et al., Phys. Rev. Lett. 70766 (1993).

[11] J. L. Krause et al., Phys. Rev.Lett. 68 3535(1992).

[12] P. B. Corkum et al., Phys. Rev. Lett. 71, 1993 (1994).

[13] M. Lewenstein et al., Phys. Rev. A 49, 2117 (1994).

[14] J. Tate et al., Phys. Rev. Lett. 98, 013901 (2007).

[15] P. Colosimo et al., Nat. Phys. 4, 386 (2008).

[16] M. V. Frolov, N. L. Manakov and A. F. Starace, Phys. Rev. Lett. 100, 173001 (2008).

[17] J. A. Pérez-Hernández, L. Roso and L. Plaja, Opt. Exp. 17, 9891 (2009). 
[18] L. E. Chipperfield et al., Phys. Rev. Lett. 6, 063003 (2009).

[19] E. Neyra et al., Eur. Phys. J. D 70, 243 (2016).

[20] E. Neyra et al., Las. Phys. Lett. 13, 115303 (2016).

[21] J. A. Pérez-Hernández et al., J. of Phys. B 13, 134004 (2009).

[22] P. Wei et al., Phys. Rev. Lett. 23, 233903 (2013).

[23] V. Strelkov, A. F. Sterjantov, N. Yu Shubin and V. T. Platonenko, J. Phys. B 39, 577-589 (2006).

[24] P. Moreno, L. Plaja, V. Malyshev and L. Roso, Phys. Rev. A 51, 4746- 4753 (1995).

[25] J. A. Pérez-Hernández, L. Roso, A. Zaïr and L. Plaja, Opt. Exp. 19, 19430-19439 (2011).

[26] J. A. Pérez-Hernández, et al., J. Phys. B 42, 134004 (2009).

[27] J. A. Pérez-Hernández, et al., Phys. Rev. Lett. 110, 053001 (2013).

[28] I. J. Kim et al., Phys. Rev. Lett. 94, 243901 (2005).

[29] M. Lara-Astasio et al., Phys. Rev. Lett. 117, 093003 (2016).

[30] X. M. Tong and C. D. Lin, J. Phys. B 38, 2593 (2005).

[31] C.K. Chui, An Introduction to Wavelets (Academic Press, New York, 1992).

[32] A. Antoine, B. Piraux and A. Maquet, Phys. Rev. A 51, 1750(R) (1995).

[33] X.M. Tong and S.I. Chu, Phys. Rev. A 61, 021802(R) (2000).

[34] J. W. Cooper, Phys. Rev. 128, 681 (1962). 